\title{
Adiponectinemia e indicadores fisio- lógicos em adolescentes obesos asmáticos e não-asmáticos
}

\section{Adiponectinemia and physiological indicators in obese asthmatics and non-asthmatics adolescents}

Fabrício Cieslak ${ }^{1}$, Nelson Augusto Rosário Filho ${ }^{2}$, Ana Claudia Kapp Titski ${ }^{3}$, Luciana da Silva Timossi ${ }^{4}$, Rodrigo Dias $^{5}$, Antonio Ramos Calixto6, Bruno Geloneze ${ }^{7}$, Neiva Leite ${ }^{8}$

\begin{abstract}
RESUMO
Introdução: Considerada um problema de saúde pública, a obesidade tem sido referida como fator de risco para o desenvolvimento da asma. Assim, o excesso de peso apresenta influencia sobre a função da musculatura lisa e induz a inflamação sistêmica das vias aéreas por intermédio das adipocitocinas. Embora a adiponectina seja considerada uma citocina de caráter anti-inflamatório, uma vez que, suas concentrações decrescem na obesidade. A literatura tem demonstrado mínimas evidências do comportamento dessa adipocitocina perante os fatores envolvidos na relação de obesidade e asma. Desse modo, o presente estudo visou analisar o comportamento das respostas de adiponectinemia e parâmetros fisiológicos em adolescentes obesos asmáticos e não-asmáticos. Métodos: Participaram 15 adolescentes obesos (seis asmáticos e nove não-asmáticos), submetidos a avaliação antropométrica, cardiorrespiratória, espirométrica e laboratorial. O diagnóstico de asma foi através de histórico clínico e questionário ISAAC, e a obesidade pelo IMC acima do percentil 95 $5^{\text {th }}$. Utilizou-se o teste de broncoprovocação com exercício físico para avaliação do broncoespasmo induzido pelo exercício (BIE). Resultados: Verificou-se diferença do \% Queda $\operatorname{VEF}_{1}(p=0,034)$ entre os obesos asmáticos e não-asmáticos. Para o obesos asmáticos, o coeficiente de determinação $\left(R^{2}\right)$ demonstrou efeito da adiponectina com a PAS $\left(r=-0,512 ; R^{2}=0,105\right)$; HDL $\left(r=+0,659 ; R^{2}=0,292\right)$, glicemia $\left(r=-0,599 ; R^{2}=0,199\right)$, glicemia120 ( $r=-$ 0,$\left.686 ; R^{2}=0,338\right)$, insulina120 $\left(r=-0,614 ; R^{2}=0,221\right), V_{2 \operatorname{máx}}$ Absoluto $\left(r=+0,518 ; R^{2}=0,086\right), V_{2 m a ́ x}$ Relativo MLG $\left(r=+0,585 ; R^{2}=0,178\right)$ e $\% \operatorname{VEF}_{1}\left(r=+0,580 ; R^{2}=0,171\right)$. Nos obesos não-asmáticos houve efeitos da adiponectina com a insulina $\left(r=-0,731 ; R^{2}=0,525\right)$, HOMA-IR $\left(r=-0,684 ; R^{2}=0,469\right)$, QUICKI $\left(r=+0,683 ; R^{2}=0,535\right)$ e $V_{2 \text { máx }}$ Relativo MLG $\left(r=+0,654 ; R^{2}=0,346\right)$. Conclusão: Verificaram-se prováveis efeitos das respostas de adiponectinemia com os parâmetros fisiológicos associados ao excesso de peso e o baixo nível de aptidão cardiorrespiratória em obesos asmáticos e não-asmáticos. Além disso, para os obesos asmáticos a adiponectinemia demonstrou um possível efeito de relação com o \%VEF .
\end{abstract}

Palavras-chave: Adiponectina. Asma. Obesidade.

1. Doutor do Colegiado de Educação Física, Universidade Federal do Vale do São Francisco, Petrolina, Pernambuco, Brasil.

2. Doutor da Unidade de Pneumologia Pediátrica do Hospital de Clínicas de Curitiba, UFPR, Curitiba, Paraná, Brasil.

3. Mestranda do Departamento de Educação Física, UFPR, Curitiba, Paraná, Brasil.

4. Doutora em Educação Física, Departamento de Educação Física, UFPR, Curitiba, Paraná, Brasil.

5. Doutorando do Departamento de Educação Física, Universidade Metodista de Piracicaba, Piracicaba, São Paulo, Brasil.

6. Doutorando em Clínica Médica, Depto de Medicina Interna, Escola de Ciências Médicas, Unicamp, Campinas/SP, Brasil.

7. Doutor em Clínica Médica, Departamento de Medicina Interna, Escola de Ciências Médicas, Unicamp, Campinas/SP, Brasil.

8. Doutora em Saúde da Criança e do Adolescente, Departamento de Educação Física, UFPR, Curitiba, Paraná, Brasil.
Correspondência:

Fabrício Cieslak, Dr. Colegiado de Educação Física, Universidade Federal do Vale do São Francisco Avenida José de Sá Maniçoba, s/n - Centro CEP: 56304-205 - Petrolina, Pernambuco, Brasil E-mail: facieslak@gmail.com

Artigo recebido em 31/01/2013 Aprovado para publicação em 19/11/2013 


\section{Introdução}

A obesidade aumentou em todas as faixas etárias, sendo considerada uma doença crônica e epidêmica. ${ }^{1} \mathrm{Na}$ população pediátrica o excesso de peso está relacionado ao estilo de vida dos adolescentes ${ }^{1}$, demonstrando uma tendência para o aumento de doenças cardiovasculares ${ }^{2}$ e possível fator de risco para os sintomas de asma. ${ }^{3,4}$

Na presença da obesidade, as propriedades do sistema respiratório são profundamente alteradas com possíveis aumentos da hiperresponsividade aérea ${ }^{5}$, sugerindo que o excesso de peso contribui para desenvolvimento de broncoespasmo induzido pelo exercício físico (BIE). ${ }^{6}$ Existem relatos de que a obesidade também está relacionada ao desencadeamento de crises de asma ao provocar alterações na mecânica respiratória e na resposta inflamatória das vias aéreas causadas pelo excesso de tecido adiposo no organismo. ${ }^{4-7}$

$\mathrm{O}$ aumento do tecido adiposo induz ao desequilíbrio na secreção de adipocitocinas, tendo papel chave no surgimento de doenças relacionadas ao estilo de vida. ${ }^{8}$ As adipocitocinas possuem numerosas funções, que incluem a regulação da saciedade, carboidratos, metabolismo lipídico e sensibilidade à insulina. ${ }^{9}$ Desse modo, há um crescente número de informações desses hormônios e, com o aumento da prevalência de obesidade e doenças associadas, a função desses peptídeos tornou-se de vital importância. ${ }^{10}$

O peptídio adiponectina tem sido identificado como uma das proteínas de secreção, referidas como adipocitocinas. ${ }^{11}$ Ela é secretada de forma abundante possui função anti-aterogênica, facilita a oxidação lipídica e estimula a ação da insulina, além de ser importante fator para controle central da homeostase energética. ${ }^{10,11,12} \mathrm{Em}$ crianças e adultos, as concentrações plasmáticas de adiponectina apresentam correlação negativa com o tecido adiposo e a resistência insulínica. ${ }^{13-17}$

Possíveis mecanismos têm sido sugeridos para a relação das adipocitocinas e asma, incluindo alterações relacionadas com a obesidade nos volumes pulmonares, inflamação sistêmica e fatores derivados dos adipócitos, que incluem a adiponectina. ${ }^{4}$ Evidências demonstram que os hormônios do tecido adiposo podem induzir alterações inflamatórias específicas e apresentar efeitos modificadores da doença em obesos asmáticos. ${ }^{7,15,17}$

As relações de adiponectina com variáveis como a massa gorda e aptidão física, não tem sido muito estudadas em crianças e adolescentes. ${ }^{13}$ Nesse contexto, o presente estudo objetivou analisar as relações comportamentais entre as respostas de adiponectina e os indicadores fisiológicos em adolescentes obesos asmáticos e não-asmáticos. A hipótese do presente estudo preconiza que os fatores associados ao excesso de peso, baixo grau de aptidão cardiorrespiratória e função pulmonar alterada estão inversamente correlacionados com a adiponectina.

\section{Materiais e Métodos}

\section{Desenho e sujeitos da pesquisa}

O delineamento do estudo é transversal e analítico. A coleta de dados foi realizada com adolescentes da Unidade de Endocrinologia Pediátrica do Hospital de Clínicas (HC de Curitiba).

O estudo foi composto por 15 adolescentes obesos (seis com a presença de asma e nove sem presença de asma), de ambos os gêneros. O número de sujeitos foi calculado com base no nível de significância de 0,05 , poder estatístico de $70 \%$ e magnitude de efeito em 0,50 , conforme classificação estabelecida na literatura ${ }^{18}$, seguido do método de recrutamento por conveniência do número de seis participantes para cada célula.

$\mathrm{O}$ protocolo de pesquisa foi fundamentado em conformidade com as diretrizes propostas na Resolução 196/96, do Conselho Nacional de Saúde, sobre pesquisas envolvendo seres humanos. $\mathrm{O}$ estudo foi aprovado no comitê de ética do Hospital de Clínicas da Universidade Federal do Paraná. (Protocolo número: $1818.235 / 2008-11$ ).

Os critérios de inclusão foram: (a) condição de participação em todas as avaliações; (b) apresentação dos termos de assentimento (adolescentes) e consentimento (pais ou responsáveis) assinados; (c) indivíduos obesos; (d) auto-relato de nenhuma infecção respiratória nas quatro semanas anteriores ao teste, baseado em exames médicos realizados antecedentes ao início das avaliações; (e) auto-relato de nenhum tratamento medicamentoso e histórico de distúrbios cardiovascular, respiratório, músculo-esquelético e/ou metabólico; (e) auto-relato de não uso de alimentos ou medicamentos com cafeína nas 12 horas precedentes a execução dos testes. Foram excluídos os indivíduos que: (a) apresentassem T4 e TSH alterados; (b) fossem classificados como pré-púberes e póspúberes na avaliação de maturação sexual. 


\section{Medidas Antropométricas, Pressão Arterial e Maturação Sexual}

Na obtenção da estatura corporal (cm) utilizouse um estadiômetro (Ayrton Corporation, resolução de $0,1 \mathrm{~cm}$ ) fixado a parede, com o indivíduo em posição ortostática, pés unidos, descalços e com a cabeça no plano horizontal de Frankfort.

A massa corporal $(\mathrm{MC})(\mathrm{kg})$ foi mensurada em balança (Filizola, resolução de $0,1 \mathrm{~kg}$ ) tipo plataforma, com o avaliado permanecendo em posição ortostática e descalço. Calculou-se o índice de massa corporal (IMC) mediante a utilização do índice de Quetelet, $\mathrm{em} \mathrm{kg} / \mathrm{cm}^{2}$ : IMC=massa corporal/estatura ${ }^{2}$, sendo classificados segundo os critérios definidos por Kuczmarski et al. ${ }^{19}$ Classificaram-se os indivíduos com o IMC entre o percentil $85^{\circ}$ e $95^{\circ} \mathrm{com}$ sobrepeso, e acima do percentil $95^{\circ} \mathrm{com}$ obesidade. O IMC foi convertido para IMC-escore Z, utilizando-se o IMC de cada indivíduo subtraído do valor correspondente ao $50^{0}$ percentil, dividido pelo desvio-padrão populacional, conforme valores disponibilizados pelo Center for Disease Control and Prevention (CDC), para cada faixa etária e sexo. ${ }^{19}$

Na determinação da circunferência abdominal (CA) foi utilizada uma fita antropométrica inelástica (resolução de $0,1 \mathrm{~cm}$ ), aplicada no ponto médio entre a crista ilíaca e a face externa da última costela, paralela ao solo, com o sujeito em pé, abdômen relaxado, braços ao longo do corpo e pés unidos.

A pressão arterial foi aferida no braço direito apoiado em nível cardíaco, utilizando-se esfigmomanômetro do tipo aneróide, previamente calibrado conforme o INMETRO. O manguito foi inflado rapidamente até $30 \mathrm{mmHg}$ acima do desaparecimento do pulso radial e desinflado a uma velocidade de 2$4 \mathrm{mmHg} /$ segundo. A pressão arterial sistólica (PAS) foi identificada pelo aparecimento dos sons e a pressão arterial diastólica (PAD) pelo seu desaparecimento (fase V de Korotkoff). Foram realizadas três medidas e consideraram-se a média das duas últimas medidas como a pressão arterial do indivíduo. ${ }^{20}$

A avaliação puberal dos indivíduos foi realizada pela auto-avaliação através de gravuras conforme os estágios maturacionais propostos por Tanner ${ }^{21}$, sendo que, as meninas foram avaliadas quanto ao desenvolvimento mamário e a pilificação pubiana e os meninos foram mensurados quanto ao tamanho testicular e a pilificação pubiana. Os indivíduos foram analisados conforme a classificação do estágios maturacionais ("Pré-púberes" - Estágio 1, "Púberes" - Estágios 2,
3 e 4 e "Pós-púberes" - Estágio 5), sendo que, foram selecionados apenas os alunos púberes. A auto-avaliação em adolescentes obesos apresenta concordância com a avaliação médica. ${ }^{22}$

\section{Composição Corporal}

A composição corporal foi avaliada pelo método da impedância bioelétrica (BIA), com o aparelho Biodynamics tetrapolar ${ }^{\circledR}$. Os indivíduos estavam em jejum de 10 a 12 horas, no período da manhã, em decúbito dorsal, após o esvaziamento vesical. Os eletrodos foram posicionados na superfície dorsal das mãos e dos pés, respectivamente próximos as articulações metacarpo-falangeanas e metatarso-falangeanas e medialmente entre as proeminências distais do rádio e da ulna, entre os maléolos tibial e fibular. Foram obtidos os valores de resistência e calculadas a massa livre de gordura e massa gorda por intermédio das equações validadas por Houtkooper et al. ${ }^{23}$

\section{Avaliação da Aptidão Cardiorrespiratória}

A aptidão cardiorrespiratória foi realizada em esteira ergométrica (marca Ecafix $700 \mathrm{X}^{\circledR}$, USA). Utilizou-se o protocolo com intensidade progressiva de acordo com a faixa etária. ${ }^{24}$

Determinou-se o consumo de oxigênio $\left(\mathrm{O}_{2}\right)$ através de um sistema de espirometria computadorizado de circuito aberto (modelo TrueOne ${ }^{\circledR} 2400$, Parvomedics, EUA). O sistema foi calibrado para $\mathrm{O}_{2} \mathrm{e} \mathrm{CO}_{2}$ usando uma concentração gasosa certificada para $\mathrm{O}_{2}$ e $\mathrm{CO}_{2}$ e para ventilação usando uma seringa de $3 \mathrm{~L}$ (modelo 5530 marca Hans Rudolph ${ }^{\circledR}$, EUA). O limiar ventilatório (LV) foi calculado individualmente e os parâmetros ventilatórios foram medidos sobre o intervalo de 15 segundos. O LV foi determinado através da combinação do método do equivalente ventilatório. ${ }^{25}$

$\mathrm{O}$ consumo máximo de oxigênio $\left(\mathrm{VO}_{2 \text { máx }}\right)$ foi representando por três unidades: 1) valores absolutos $\left(1 . \mathrm{min}^{-1}\right)$, representando por $\left.\mathrm{VO}_{2 \text { máx }} 1 .(\mathrm{min})^{-1} ; 2\right)$ relativo a massa corporal $\left(\mathrm{ml} . \mathrm{kg} \cdot \mathrm{min}^{-1}\right)$, representado por $\left.\mathrm{VO}_{2 \text { máx }} \mathrm{ml} .(\mathrm{kg} . \mathrm{min})^{-1} ; 3\right)$ relativo a massa livre de gordura (ml.Kg.MLG.min ${ }^{-1}$ ), representado por $\mathrm{VO}_{2 \text { máx }}$ ml.(kg. MLG. min $)^{-1}$.

A freqüência cardíaca (FC) (em bpm) foi mensurada continuamente durante a realização do teste, através da utilização de cardiofrequencímetro (marca Polar ${ }^{\circledR}$, modelo S625X, Kempele, Finlândia).

\section{Teste de Função Pulmonar}

Os participantes foram orientados para não ingerir café, chá ou refrigerante com cafeína duas ho- 
ras antes da avaliação, a suspender o uso de broncodilatadores de ação curta e longa 12 horas antes e os anti-histamínicos de ação curta e longa, respectivamente, 48 horas e cinco dias antes da avaliação. Os avaliados não poderiam apresentar sintomas de infecção viral de vias aéreas superiores nas últimas quatro semanas e não estar em crise de asma. Aplicou-se o questionário International Study of Asthma and Allergies in Childhood (ISAAC) ${ }^{6}$ para identificação dos fatores históricos e atuais do desenvolvimento de asma e alergia. Posteriormente, efetuou-se a prova de função pulmonar para confirmação do diagnóstico de asma. $^{26}$

A função pulmonar foi mensurada por espirômetro (marca Microlab $2000^{\circledR}$ ), em posição sentada e com o uso de clipe nasal. As variáveis pulmonares mensuradas foram a Capacidade Vital Forçada (CVF) e o volume expiratório forçado no primeiro segundo $\left(\mathrm{VEF}_{1}\right)$, em litros.

Realizaram-se até oito manobras espirométricas, seleci$\%$ Queda $_{\text {máx }} \mathrm{VEF}_{1}=\left(\left(\mathrm{VEF}_{1 \text { pós-exercício mais baixo }}-\mathrm{VEF}_{1 \text { pré-exercício }}\right) \times 100\right) / \mathrm{VEF}_{1 \text { pré-exercício }}$. onando-se as três manobras aceitáveis e reprodutíveis, com diferença máxima de 5\%. Em seguida, após análise das três curvas, foi escolhida aquela com maiores valores do $\mathrm{VEF}_{1}$ e CVF. Calcularam-se os percentuais dos valores preditos do $\mathrm{VEF}_{1}$ e CVF para a idade e sexo, conforme Polgar; Promodhat ${ }^{27}$ e a relação $\mathrm{VEF}_{1} / \mathrm{CVF}$.

Os protocolos de função pulmonar foram realizados em dias de avaliações programadas aos pacientes, sempre no período da tarde (14:00 as 17:00 horas) e com ambiente controlado (temperatura entre $20^{\circ}$ a $25^{0}$ Celsius e umidade menor que 50\%). Antes dos testes foram explicadas as técnicas de manobras necessárias a realização do teste de função pulmonar, para ter resultados reprodutivos, seguindo as diretrizes para teste de função pulmonar ${ }^{28}$ e não apresentando $\mathrm{VEF}_{1}$ menor que $75 \%$ no dia do teste.

A prova de exercício físico foi realizada em esteira ergométrica (marca Ecafix $700 \mathrm{X}^{\circledR}$, USA) utilizando o protocolo proposto pela American Thoracic Society $^{28}$ que consistia em caminhar/correr durante oito minutos, numa intensidade superior a $85 \%$ da freqüência cardíaca máxima $\left(\mathrm{FC}_{\text {máx }}\right)$.

Para atingir a intensidade prevista a velocidade e a inclinação da esteira foram ajustadas. A inclinação ficou estabelecida em $10 \%$ e a velocidade estimada, conforme estudo anterior. ${ }^{6}$

A frequiência cardíaca foi monitorada por mo- nitor cardíaco (marca Polar ${ }^{\circledR}$, modelo S625X, Kempele, Finlândia). Os testes foram realizados no período da tarde, das 14 às 17 horas e o ambiente de execução foi controlado em 20 a $25^{\circ} \mathrm{C}$ de temperatura e a umidade do ar abaixo de $50 \%$.

O volume expiratório forçado no primeiro segundo $\left(\mathrm{VEF}_{1}\right)$ foi mensurado, em litros, nos 3, 5, 10, 15 e 30 minutos após o exercício físico. O BIE foi considerado positivo para uma redução do $\mathrm{VEF}_{1}$ igual ou superior a $10 \%$ ao valor pré-exercício. ${ }^{6}$

Além das mensurações dos volumes expiratórios forçados no primeiro segundo $\left(\mathrm{VEF}_{1}\right)$ nos testes de broncoprovocação, após o exercício físico, foram calculados em ambos os testes a queda percentual máxima do $\operatorname{VEF}_{1}\left(\%\right.$ Queda $\left._{\text {máx }} \mathrm{VEF}_{1}\right)$, utilizando o cálculo da diminuição percentual do $\mathrm{VEF}_{1}$ pós-exercício em relação ao valor pré-exercício pela seguinte equação:

\section{Exames Laboratoriais}

As amostras sanguíneas foram coletadas no período da manhã, após 12 horas de jejum, para realização de hemograma e dosagens de glicose, insulina, colesterol total (CT), lipoproteína de alta densidade (HDL-C), lipoproteína de baixa densidade (LDL-C) e triglicérides (TG). Em seguida, os adolescentes ingeriram uma solução contendo 1,75 gramas de glicose/ $\mathrm{kg}$, sendo que, após 120 minutos foram coletadas amostras para dosagem da Glicemia120 e Insulina120. As mensurações foram realizadas nas sessões de Bioquímica, Hematologia e Dosagens Hormonais do Serviço de Análises Clínicas do HC da Universidade Federal do Paraná (UFPR).

As concentrações plasmáticas de CT, TG e de HDL-C foram determinadas em $\mathrm{mg} / \mathrm{dl}$, através de teste colorimétrico enzimático (CHOD-PAP) (Laboratório Merck, Darmstadt, Alemanha; Laboratório Roche, Indianápolis, IN, EUA). O LDL-C foi calculado pela equação de Friedwald et al. (29), em mg/dl: $\mathrm{LDL}=\mathrm{CT}-(\mathrm{HDL}+\mathrm{TG} / 5)$. Foram considerados como referência os valores para lipídios apresentados na I Diretriz de Prevenção da Aterosclerose na Infância e Adolescência. ${ }^{30}$

Os níveis de glicemia foram determinados com o método enzimático (Glicose Oxidase - Labtest) e 
foram baseados na American Diabetes Association. ${ }^{30} \mathrm{~A}$ insulina foi dosada pela técnica de quimiluminescência por imunoensaio imunométrico em ${ }_{\mathrm{u}} \mathrm{u} / \mathrm{ml}$, utilizando como referência o ponto de corte recomendado na I Diretriz de Prevenção da Aterosclerose na Infância e Adolescência. ${ }^{31}$ Para o cálculo da resistência a insulina utilizou-se a Homeostasis Model Assessment (HOMA-IR) descrito por Matthews et al. ${ }^{32}$ Para avaliação da sensibilidade a insulina foi utilizada o Quantitative Insulin Sensivity Check Index (QUICKI) descrito por Katz et al. ${ }^{33}$ A adiponectina foi dosada utilizando-se do kit ELISA da B-Bridge International, Inc (Sunnyvale, CA, EUA). A variabilidade intraensaio e interensaio da adiponectina foi de 1,92 e $8,1 \%$, respectivamente.

\section{Análise Estatística}

Utilizaram-se medidas descritivas para caracterização dos participantes e testes apropriados conforme a literatura. ${ }^{19}$ A normalidade dos dados foi realizada pelo teste de Shapiro-Wilk. Os dados que não apresentaram distribuição normal foram transformados em valores de logaritmo natural $(\log n)$ para normalização. Após este procedimento aplicou-se o teste $\mathrm{T}$ para amostras independentes na comparação das variáveis entre os grupos. Para comparação das variáveis espirométricas $\left(\mathrm{VEF}_{1}\right.$ basal (\% pred), $\mathrm{CVF}$ basal (\% pred), $\mathrm{VEF}_{1} / \mathrm{CVF}$ basal e \% Queda ${ }_{\text {máx }} \mathrm{VEF}_{1}$ ) que apresentavam características percentuais utilizouse o teste $\mathrm{Z}$ de Kolmogorov-Smirnov. Para analisar as possíveis relações da adiponectina com os parâmetros fisiológicos utilizou-se a correlação de Pearson. Em seguida, para as análises correlacionais de tamanho de efeito grande ${ }^{18}$ foi aplicada a regressão linear para predizer, através do coeficiente de regressão (â) e do coeficiente de determinação $\left(\mathrm{R}^{2}\right)$, o comportamento de variação da adiponectina em relação a variáveis fisiológicas. Esses procedimentos foram realizados dividindo-se os participantes em dois grupos, pois o intuito do estudo foi verificar o comportamento da adiponectina sobre as outras variáveis em obesos asmáticos e não-asmáticos. Para verificar a magnitude das análises dos testes comparativos foram calculados os tamanhos de efeito " $d$ ", enquanto para os testes correlacionais utilizou-se o tamanho de efeito " $r$ ", conforme propostos pela literatura. ${ }^{18}$ Para os testes de correlações, o tamanho de efeito é referido como o grau em que o fenômeno de relação está presente na amostra elevando o poder da análise, ou seja, pode-se considerar como efeito pequeno $(\mathrm{r}=0,10)$, efeito mo- derado $(\mathrm{r}=0,30)$ ou efeito grande $(\mathrm{r}=0,50) .{ }^{18}$ Os procedimentos estatísticos foram realizados mediante a utilização do Statistical Package for the Social Sciences (SPSS, versão 20.0) for Windows, com nível de significância fixado em $\mathrm{p}<0,05$.

\section{Resultados}

Foram estudados 15 adolescentes (nove meninas e seis meninos), cujas características antropométricas e os parâmetros laboratoriais das avaliações iniciais são apresentadas na Tabela 1, conforme a presença ou não de asma. Na avaliação dos 15 adolescentes, verificou-se que nove não apresentam o diagnóstico de asma e seis demonstram a presença. As médias das variáveis antropométricas estão acima dos valores recomendados para adolescentes eutróficos. Não foram encontradas diferenças significativas entre os grupos para as variáveis antropométricas e parâmetros laboratoriais, porém alguns indivíduos não estão dentro dos valores recomendáveis para adolescentes, na glicemia (20\%), insulinemia (73\%), CT (13\%), HDL-C (40\% dos sujeitos), LDL-C (7\%), triglicérides $(47 \%)$, glicemia $(20 \%)$, insulina $(73,3 \%)$, HOMA-IR (66,7\%) e QUICKI $(53,3)$. A adiponectina apresentou valores de baixas concentrações para ambos os grupos e não foram verificadas diferenças significativas.

Os valores das variáveis cardiorrespiratórias e espirométricas dos indivíduos avaliados constam na Tabela 2. Não foram identificadas diferenças para as variáveis cardiorrespiratórias entre os indivíduos asmáticos e não-asmáticos. Para as variáveis espirométricas, foram encontradas diferenças significativas $(\mathrm{p}=0,034)$ com magnitude de efeito moderado $(d=$ $0,55)$ para \% Queda $V_{E F}$ entre os indivíduos asmáticos e não-asmáticos. A broncoprovocação por exercício físico foi positiva em $40 \%$ dos sujeitos $(66,7 \%$ em asmáticos e 23,3\% em não-asmáticos).

As correlações com tamanho de efeito grande das características antropométricas, cardiorrespiratórias, espirométricas e parâmetros laboratoriais com adiponectina dos adolescentes obesos asmáticos são apresentadas na Figura 1. Para os adolescentes obesos asmáticos não foram verificadas correlações significativas. Porém, constataram-se tendências de relações para tamanho de efeito grande da adiponectina com a PAS ( $r=-0,512 ; \mathrm{p}=0,299)$, HDL $(\mathrm{r}=+0,659$; $\mathrm{p}=0,155)$; glicemia $(\mathrm{r}=-0,599 ; \mathrm{p}=0,209)$; glicemia 120 $(\mathrm{r}=-0,686 ; \mathrm{p}=0,132)$; insulina $120(\mathrm{r}=-0,614 ; \mathrm{p}=0,195)$; 


\section{Tabela 1}

Variáveis antropométricas e parâmetros laboratoriais dos adolescentes.

\begin{tabular}{|c|c|c|c|c|c|c|c|c|c|c|}
\hline \multirow{2}{*}{$\begin{array}{l}\text { Variáveis } \\
\text { Idade (meses) }\end{array}$} & \multicolumn{3}{|c|}{$\begin{array}{c}\text { Obeso } \\
\text { Asmático } \\
(n=6)\end{array}$} & \multicolumn{3}{|c|}{$\begin{array}{c}\text { Obeso } \\
\text { Não-asmático } \\
(\mathbf{n}=9)\end{array}$} & \multicolumn{3}{|c|}{$\begin{array}{c}\text { Total } \\
(\mathrm{n}=15)\end{array}$} & \multirow{2}{*}{$\begin{array}{r}\begin{array}{r}\text { Tamanho } \\
\text { de Efeito } \\
(\boldsymbol{d})\end{array} \\
0,33\end{array}$} \\
\hline & 150,3 & \pm & 27,0 & 166,8 & \pm & 21,4 & 160,2 & \pm & 24,3 & \\
\hline Idade (anos) & 12,5 & \pm & 2,2 & 13,9 & \pm & 1,7 & 13,3 & \pm & 2,0 & 0,33 \\
\hline $\mathrm{MC}(\mathrm{kg})$ & 81,7 & \pm & 17,5 & 80,7 & \pm & 11,9 & 81,1 & \pm & 13,8 & 0,03 \\
\hline Estatura $(\mathrm{cm})$ & 159,7 & \pm & 9,9 & 163,3 & \pm & 7,5 & 161,9 & \pm & 8,4 & 0,16 \\
\hline $\operatorname{IMC}\left(\mathrm{kg} / \mathrm{m}^{2}\right)$ & 31,8 & \pm & 5,3 & 30,1 & \pm & 3,4 & 30,8 & \pm & 4,2 & 0,12 \\
\hline IMC escore-Z & 4,1 & \pm & 1,7 & 3,1 & \pm & 1,1 & 3,5 & \pm & 1,4 & 0,27 \\
\hline $\mathrm{CA}(\mathrm{cm})$ & 103,3 & \pm & 14,2 & 98,5 & \pm & 8,5 & 100,5 & \pm & 10,9 & 0,18 \\
\hline$\%$ de Gordura & 38,4 & \pm & 4,8 & 36,5 & \pm & 5,1 & 37,3 & \pm & 4,9 & 0,15 \\
\hline $\mathrm{MG}(\mathrm{kg})$ & 31,7 & \pm & 9,5 & 29,4 & \pm & 6,9 & 30,3 & \pm & 7,8 & 0,09 \\
\hline MLG (kg) & 50,2 & \pm & 10,1 & 50,7 & \pm & 7,2 & 50,5 & \pm & 8,2 & 0,06 \\
\hline PAS (mmHg) & 110,3 & \pm & 16,2 & 100,2 & \pm & 13,1 & 104,2 & \pm & 14,7 & 0,35 \\
\hline $\mathrm{PAD}(\mathrm{mmHg})$ & 72,3 & \pm & 14,3 & 68,6 & \pm & 10,3 & 70,1 & \pm & 11,7 & 0,18 \\
\hline $\mathrm{CT}(\mathrm{mg} / \mathrm{dL})$ & 147,1 & \pm & 12,3 & 152,4 & \pm & 29,5 & 150,3 & \pm & 23,6 & 0,12 \\
\hline HDL-C (mg/dL) & 41,5 & \pm & 6,8 & 42,6 & \pm & 8,0 & 42,2 & \pm & 7,3 & 0,04 \\
\hline LDL-C (mg/dL) & 81,3 & \pm & 7,0 & 85,7 & \pm & 21,9 & 84,0 & \pm & 17,2 & 0,23 \\
\hline Triglicérides(mg/dL) & 122,3 & \pm & 47,5 & 119,8 & \pm & 61,1 & 120,8 & \pm & 54,2 & 0,12 \\
\hline Glicemia (mg/dL) & 94,5 & \pm & 6,7 & 95,4 & \pm & 7,4 & 95,0 & \pm & 6,9 & 0,15 \\
\hline Glicemia120(mg/dL) & 95,6 & \pm & 21,0 & 99,8 & \pm & 10,7 & 98,2 & \pm & 15,1 & 0,12 \\
\hline Insulina $(\mu \mathrm{UI} / \mathrm{mL})$ & 23,3 & \pm & 14,9 & 18,8 & \pm & 7,4 & 20,6 & \pm & 10,7 & 0,09 \\
\hline Insulina120( $\mu \mathrm{UI} / \mathrm{mL})$ & 55,8 & \pm & 43,0 & 42,8 & \pm & 27,1 & 48,0 & \pm & 33,5 & 0,12 \\
\hline HOMA-IR & 5,57 & \pm & 3,81 & 4,40 & \pm & 1,66 & 4,87 & \pm & 2,67 & 0,06 \\
\hline QUICKI & 0,31 & \pm & 0,03 & 0,31 & \pm & 0,02 & 0,31 & \pm & 0,02 & 0,06 \\
\hline Adiponectina $(\mu \mathrm{g} / \mathrm{mL})$ & 5,86 & \pm & 1,58 & 4,56 & \pm & 1,78 & 5,08 & \pm & 1,77 & 0,34 \\
\hline
\end{tabular}

$* p<0,05$ 
Tabela 2

Variáveis cardiorrespiratórias e espirométricas dos adolescentes

\begin{tabular}{|c|c|c|c|c|c|c|c|c|c|}
\hline \multirow{2}{*}{$\frac{\text { Variáveis }}{\mathrm{FC}_{\text {rep }}(\mathrm{bpm})}$} & \multicolumn{2}{|c|}{$\begin{array}{c}\text { Obeso } \\
\text { Asmático } \\
(n=6)\end{array}$} & \multicolumn{3}{|c|}{$\begin{array}{c}\text { Obeso } \\
\text { Não-asmático } \\
(\mathbf{n}=9)\end{array}$} & \multicolumn{3}{|c|}{$\begin{array}{c}\text { Total } \\
(\mathrm{n}=15)\end{array}$} & \multirow{2}{*}{$\begin{array}{r}\begin{array}{r}\text { Tamanho } \\
\text { de Efeito } \\
(\boldsymbol{d})\end{array} \\
0,37\end{array}$} \\
\hline & 85,3 & $\pm 12,5$ & 76,8 & \pm & 7,1 & 80,2 & \pm & 10,1 & \\
\hline $\mathrm{FC}_{\text {máx }}(\mathrm{bpm})$ & 192,6 & $\pm 11,1$ & 196,5 & \pm & 8,4 & 195,0 & \pm & 9,4 & 0,20 \\
\hline $\mathrm{VO}_{2 \text { máx }}$ Absoluto 1. (min) $)^{-1}$ & 2,82 & 0,69 & 3,07 & \pm & 0,46 & 2,97 & \pm & 0,55 & 0,43 \\
\hline $\mathrm{VO}_{2 \text { máx }}$ Relativo ml . (kg.min $)^{-1}$ & 32,7 & 7,0 & 37,0 & \pm & 6,9 & 35,3 & \pm & 7,0 & 0,33 \\
\hline $\mathrm{VO}_{2 \max }$ Relativo MLGml.(kg.MLG.min) $)^{-1}$ & 52,6 & 7,1 & 58,5 & \pm & 7,8 & 56,2 & \pm & 7,9 & 0,37 \\
\hline $\mathrm{VEF}_{1}$ basal $(\mathrm{L} / \mathrm{min})$ & 2,80 & 0,74 & 3,11 & \pm & 0,51 & 2,99 & \pm & 0,60 & 0,37 \\
\hline $\mathrm{VEF}_{1}$ basal $(\%$ pred $)$ & 90,5 & 9,9 & 95,2 & \pm & 14,0 & 91,0 & \pm & 12,4 & 0,06 \\
\hline CVF basal (L/min) & 3,42 & $\pm \quad 1,04$ & 3,63 & \pm & 0,60 & 3,55 & \pm & 0,78 & 0,18 \\
\hline CVF basal (\% pred) & 103,4 & $\pm \quad 13,9$ & 104,1 & \pm & 13,2 & 103,8 & \pm & 13,0 & 0,02 \\
\hline $\mathrm{VEF}_{1} / \mathrm{CVF}$ basal & 82,9 & 7,1 & 86,1 & \pm & 7,6 & 84,6 & \pm & 7,4 & 0,18 \\
\hline $\mathrm{VEF}_{1}$ Queda $_{\text {máx }}(\mathrm{L} / \mathrm{min})$ & 2,29 & $\pm \quad 0,83$ & 2,84 & \pm & 0,52 & 2,67 & \pm & 0,70 & 0,43 \\
\hline$\%$ Queda $_{\text {máx }} \mathrm{VEF}_{1}$ & & $1,21 *$ & & $-7,51$ & & & 8,28 & & 0,55 \\
\hline
\end{tabular}

$\mathrm{VO}_{2 \text { máx }}$ Absoluto $(\mathrm{r}=+0,518 ; \mathrm{p}=0,292) ; \mathrm{VO}_{2 \text { máx }}$ Relativo MLG ( $\mathrm{r}=+0,585 ; \mathrm{p}=0,223)$ e $\% \mathrm{VEF}_{1}$ Predito $(r=+0,580 ; p=0,227)$. A correlação de efeito moderado da adiponectina ocorreu perante as seguintes variáveis: PAD ( $\mathrm{r}=-0,370 ; \mathrm{p}=0,470)$, FCrep $(\mathrm{r}=-0,306$; $\mathrm{p}=0,555) ; \mathrm{VO}_{2 \text { máx }}$ Relativo $(\mathrm{r}=+0,464 ; \mathrm{p}=0,353), \mathrm{VEF}_{1}$ $(\mathrm{r}=+0,473 ; \mathrm{p}=0,343)$ e $\mathrm{CVF}(\mathrm{r}=+0,344 ; \mathrm{p}=0,504)$.

O coeficiente de determinação da regressão demonstrou que o efeito da adiponectina em adolescentes obesos asmáticos, sobre as variáveis fisiológicas com tamanho de efeito grande, pode ser representando da seguinte forma: PAS (10\%), HDL (29\%), glicemia (19,9\%), glicemia $120(33,8 \%)$, insulina 120 $(22,1 \%), \mathrm{VO}_{2 \operatorname{máx}}$ Absoluto $(8,6 \%) ; \mathrm{VO}_{2 \text { máx }}$ Relativo MLG $(17,8 \%)$ e $\% \mathrm{VEF}_{1}$ Predito $(17,1 \%)$.

As análises correlacionais dos adolescentes obesos não-asmáticos com tamanho de efeito grande, das variáveis antropométricas, cardiorrespiratórias, espirométricas e parâmetros laboratoriais com adiponectina são apresentadas na Figura 2. Os ado- lescentes obesos não-asmáticos demonstraram correlações significativas e com tamanho de efeito grande da adiponectina com a insulina $(\mathrm{r}=-0,731 ; \mathrm{p}=0,025)$, HOMA-IR ( $r=-0,684 ; \mathrm{p}=0,042)$ e QUICKI ( $\mathrm{r}=+0,683$; $\mathrm{p}=0,043)$. Verificou-se ainda, tendência de relação para tamanho de efeito grande da adiponectina com a $\mathrm{VO}_{2 \text { máx }}$ Relativo MLG ( $\left.\mathrm{r}=+0,654 ; \mathrm{p}=0,056\right)$. As relações de efeito moderado da adiponectina foram verificadas com a: $\mathrm{MG}(\mathrm{r}=-0,402 ; \mathrm{p}=0,284)$; $\mathrm{PAD}(\mathrm{r}=-$ $0,316 ; \mathrm{p}=0,408)$, Glicemia $(\mathrm{r}=-0,312 ; \mathrm{p}=0,414)$; Glicemia $120(\mathrm{r}=-0,489 ; \mathrm{p}=0,182), \mathrm{VO}_{2 \text { máx }}$ Relativo $(\mathrm{r}=+0,455 ; \mathrm{p}=0,218), \mathrm{VEF}_{1}(\mathrm{r}=+0,310 ; \mathrm{p}=0,417)$ e CVF $(r=+0,396 ; p=0,291)$.

Para análise do coeficiente de determinação verificou-se que o efeito da adiponectina em adolescentes obesos não-asmáticos, sobre componentes fisiológicos com tamanho de efeito grande, pode ser descrito do seguinte modo: insulina $(52,5 \%)$, HOMA-IR (46,9\%), QUICKI $(53,5 \%)$ e $V_{2 \text { máx }}$ Relativo MLG $(34,6 \%)$. 

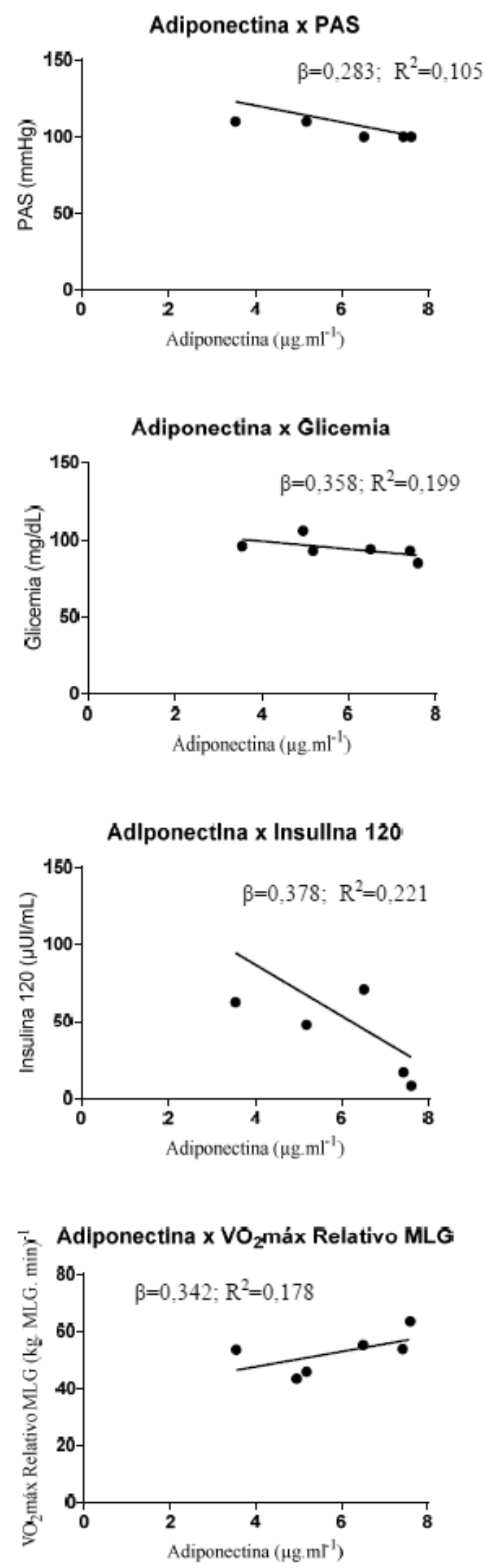

Adlponectlna $\times \mathrm{HDL}$
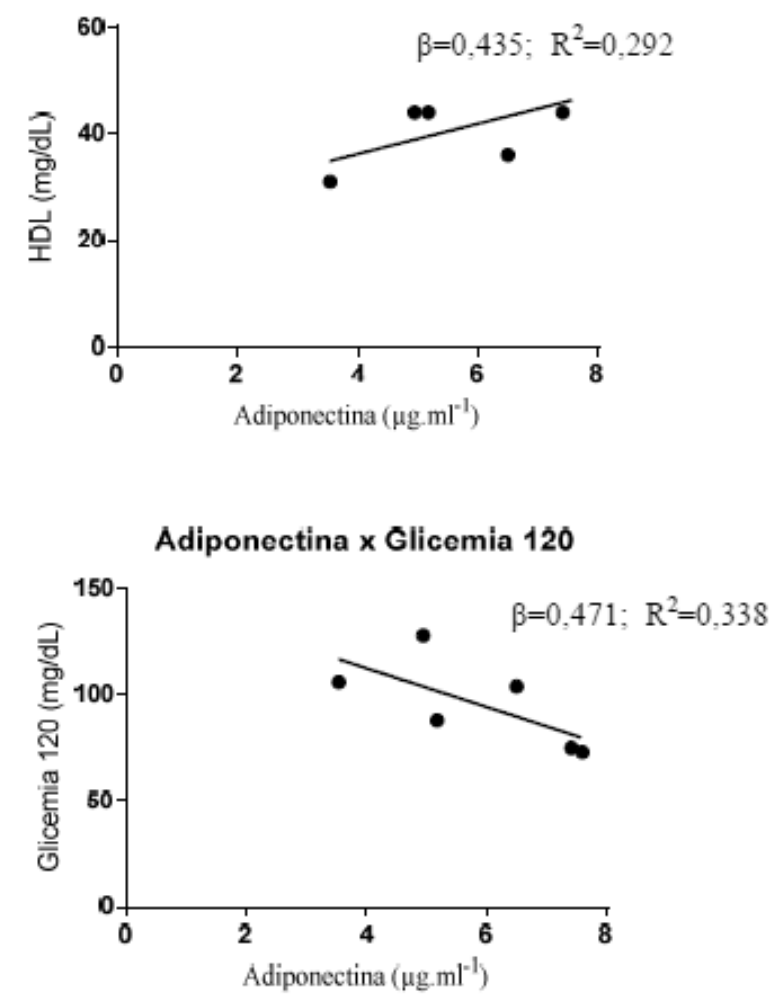

Adiponectina $\times \mathrm{VO}_{2}$ máx Absoluto

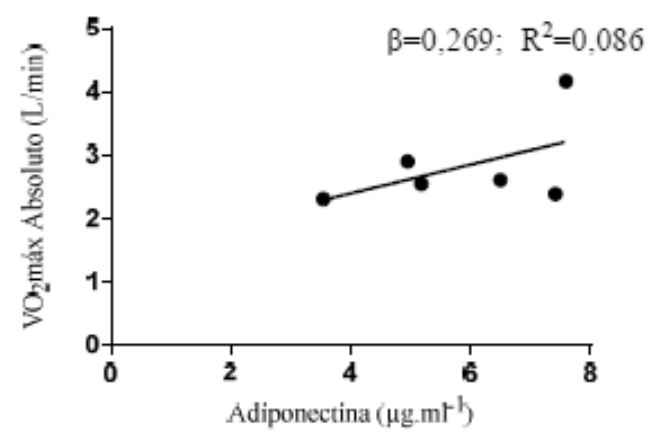

Adiponectina $x \operatorname{VEF}_{\mathbf{1}}(\%$ Predito $)$

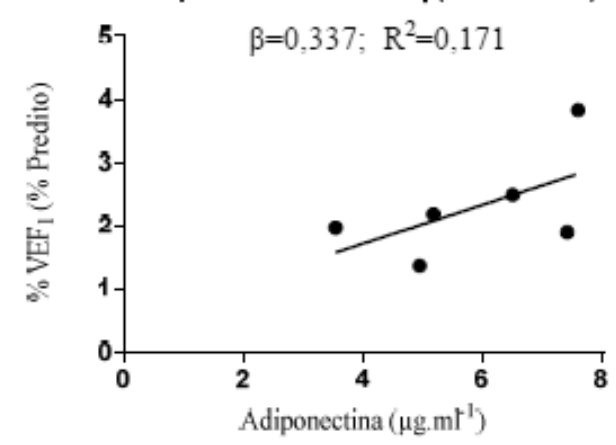

Figura 1: Correlações da adiponectina e variáveis dos adolescentes obesos asmáticos. * $\mathrm{p}<0,05$ 

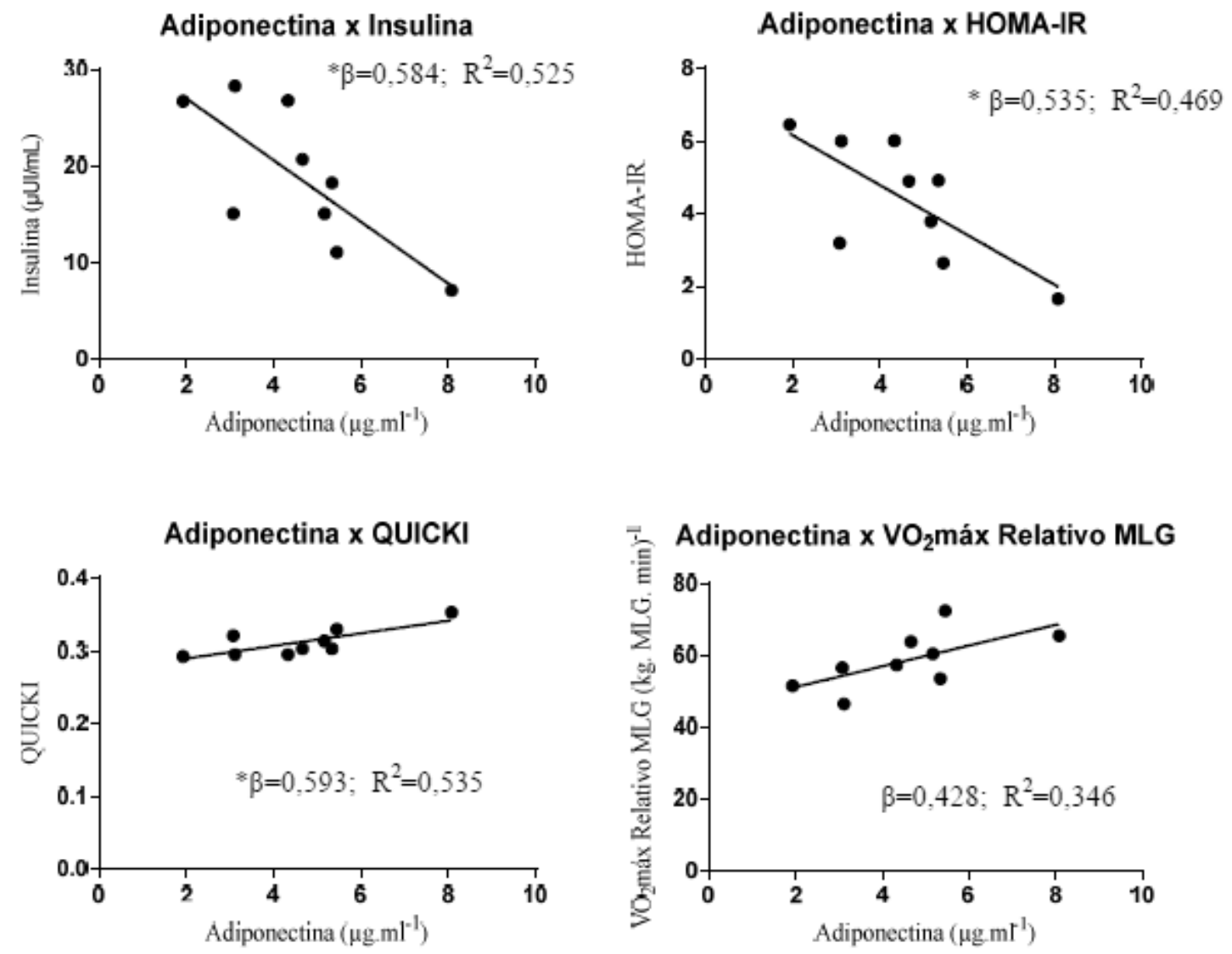

Figura 2: Correlações da adiponectina e variáveis dos adolescentes obesos não-asmáticos.

\section{Discussão}

A relação entre asma e obesidade tem sido objeto de vários estudos $(4,5,6,16)$, principalmente porque as duas situações clínicas estão atingindo proporções epidêmicas, indicando que as mudanças ambientais e sociais do mundo moderno estão envolvidas na gênese, além das interações entre ambas as doenças. ${ }^{3}$ A literatura tem indicado que a prevalência de asma é maior em obesos quando comparados a indivíduos eutróficos. ${ }^{4}$

Os mecanismos de obesidade e asma ainda não estão bem estabelecidos, as possíveis relações podem ser influenciadas pelo efeito da obesidade sobre a mecânica ventilatória, inflamação sistêmica, hormônios de regulação do metabolismo energético, comorbidades e etiologias comuns. ${ }^{4}$ A presente pesquisa indicou que os adolescentes obesos asmáticos e não-asmáticos não apresentam diferenças significativas entre as variáveis antropométricas, cardiorrespiratórias e laboratoriais. Entretanto, apesar de nossos resulta- dos, as pesquisas em crianças e adultos têm demonstrado que a obesidade antecede a asma, e que o risco relativo de incidência da asma aumenta com o excessivo índice de massa corporal. ${ }^{4,16}$

$\mathrm{Na}$ análise das variáveis espirométricas, os valores do nosso estudo indicaram maior ocorrência de $\%$ Queda $_{\text {máx }} \mathrm{VEF}_{1}$ em obesos asmáticos comparados aos obesos não-asmáticos. Em estudo realizado por Cieslak et al. ${ }^{6}$ avaliou-se o broncoespasmo induzido pelo exercício em adolescentes, que incluíam obesos asmáticos e obesos não-asmáticos, com valores demonstrando maior severidade dos indicadores em adolescentes obesos asmáticos comparados aos obesos que não apresentam diagnóstico de asma. Esses resultados sugerem que o asmático obeso permaneça mais tempo de quadro de broncoconstrição por broncoespasmo induzido pelo exercício que os não-obesos. ${ }^{3}$

Considerada uma molécula chave de proteção inflamatória, a adiponectina é a única em que a concentração plasmática decresce na obesidade. ${ }^{11}$ Os mecanismos que estão relacionados ao tecido adiposo 
com processos patológicos, como a adiponectina, têm sido pouco mensurados em estudos com populações pediátricas. ${ }^{13}$ Em nossa pesquisa, apesar dos obesos asmáticos demonstrarem valores médios superiores aos obesos não-asmáticos, não foram verificadas diferenças significativas para os valores de adiponectina entre os grupos. Esses resultados corroboram com outra pesquisa que avaliou as adipocitocinas e manifestações de asma em crianças e não verificaram diferenças significativas entre os grupos para a adiponectinemia. ${ }^{7}$ Embora ambas pesquisas indiquem que os obesos asmáticos e não-asmáticos não apresentam diferenças significativas para os valores de adiponectina, novas pesquisas são necessárias para verificar o real comportamento em obesos e asmáticos, já que a literatura relata que as concentrações séricas dessa variável no obeso podem induzir ao desenvolvimento de hiperresponsividade das vias aéreas. ${ }^{15}$

Na presente pesquisa, não houveram relações significativas das variáveis fisiológicas com a adiponectina em adolescentes obesos asmáticos. Entretanto, as análises do coeficiente de determinação podem exemplificar o comportamento de determinadas variáveis, como o efeito negativo de $10 \%$ da adiponectina com a PAS. Uma resposta plausível para esse achado refere-se ao fato de que, o aumento da pressão arterial está relacionado a níveis elevados de endotelina, um potente broncoconstritor. A literatura aborda ainda que, o aumento da pressão arterial pode causar insuficiência cardíaca seguida de congestão pulmonar, que poderia amplificar o edema brônquico periférico e, consequentemente, ocasionar um agrave maior do desacoplamento das vias respiratórias. ${ }^{4}$

A literatura tem demonstrado a influência da adiponectinemia sobre variáveis metabólicas em adolescentes obesos. ${ }^{7,8,11,13,15,16}$ Nesse sentido, essas evidências corroboram com nossos achados que apresentaram relações significativas da adiponectinemia com as variáveis de insulinemia nos adolescentes obesos não-asmáticos, além de possíveis efeitos de HDL, glicemia, glicemia 120 e insulina 120 em obesos asmáticos. Desse modo, o reverso da hipoadiponectinemia em obesos asmáticos e não-asmáticos pode ser fator de proteção para doenças da síndrome metabólica. ${ }^{11}$ Além disso, as respostas das variáveis de glicemia com adiponectinemia em adolescentes obesos asmáticos verificados podem fornecer subsídios para explicar a relação da obesidade com a asma, uma vez que, o diabetes tipo 2 é um complicador comum da obesidade. ${ }^{4}$

Os achados referentes as correlações de adi- ponectinemia e variáveis cardiorrespiratórias detectados neste estudo foram verificadas em outras evidências. ${ }^{13,34}$ Para a pesquisa de Nemet et al $^{13}$, que avaliou o efeito das concentrações de adiponectina com o consumo de oxigênio em 30 adolescentes saudáveis houve relação moderada e significativa entre as variáveis. Outra análise com homens acima de 35 anos revelou que a adiponectina está positivamente correlacionada ao consumo de oxigênio. ${ }^{34} \mathrm{Na}$ presente pesquisa, as correlações das variáveis cardiorrespiratórias com a adiponectina demonstraram tamanho de efeito grande sem valores significativos. Desse modo, os indicadores sugerem que um condicionamento cardiorrespiratório adequado pode elevar as concentrações de adiponectinemia. É importante ressaltar que, a ausência de significância das análises estatísticas desse estudo podem ser explicadas pelo número amostral reduzido dos adolescentes obesos asmáticos e não asmáticos, ou seja, um número maior de participantes poderia demonstrar um efeito significativo dessas variáveis nos adolescentes. Nesse sentido, o estudo do comportamento das adipocitocinas com as variáveis cardiorrespiratórias e fatores relacionados a obesidade e asma em adolescentes são necessários para fornecer subsídios válidos ao avanço do conhecimento clínico em novas estratégias terapêuticas para o tratamento nessa população. ${ }^{4}$

As evidências da literatura apresentam lacunas no comportamento da adiponectinemia com as variáveis espirométricas. Na presente pesquisa, verificouse um possível efeito da adiponectina sobre o $\% \mathrm{VEF}_{1}$ em adolescentes obesos asmáticos, para as outras variáveis de função pulmonar não foram encontradas relações significativas da adiponectina em adolescentes obesos asmáticos e não-asmáticos. Embora algumas evidências prévias ${ }^{8,15}$ não tenham demonstrado relação da adiponectina com a função pulmonar, um estudo revelou que a obesidade está associada a inflamação sistêmica, causando alterações hormonais do tecido adiposo que podem induzir a mudanças inflamatórias específicas em obesos asmáticos. ${ }^{17}$

$\mathrm{O}$ presente estudo deve ser interpretado com cautela diante de alguns fatores limitantes. O delineamento amostral de seleção não-aleatória dos sujeitos com características étnicas próprias pode ter ocasionado um viés de seleção. $\mathrm{O}$ baixo número de participantes pode ter influenciado nos resultados das correlações, principalmente em variáveis com valores de magnitude de efeito moderado que poderiam apresentar resultados mais consistentes na explanação dos 
efeitos da relação obesidade e asma, além de elevar o poder estatístico do cálculo amostral. Embora o estudo tenha explorado apenas algumas variáveis pulmonares, a presente pesquisa corrobora com os subsídios da literatura em relação a associação de determinadas variáveis. A ausência da medida de fluxo expiratório forçado entre 25 e $75 \%\left(\mathrm{FEF}_{25 \%-75 \%}\right)$, apesar de limitante, justifica-se pelo fato de que essa medida não apresenta grau significativo de influência em estudos com obesos. ${ }^{35,36}$

\section{Conclusão}

Verificou-se que os adolescentes obesos asmáticos demonstraram maior índice de \% Queda ${ }_{\text {máx }} \mathrm{VEF}_{1}$ em comparação aos obesos não-asmáticos. Houve efeito considerado grande das respostas de adiponec- tinemia com os indicadores fisiológicos associados a obesidade em asmáticos e não-asmáticos, evidenciando que os parâmetros de síndrome metabólica e o baixo nível de aptidão cardiorrespiratória possivelmente estão envolvidos no processo inflamatório. Além disso, a adiponectinemia demonstrou um provável efeito de associação com o \% $\mathrm{VEF}_{1}$ nos obesos asmáticos.

\section{Agradecimentos}

Os autores da pesquisa agradecem a Coordenação de Aperfeiçoamento de Pessoal de Nível Superior (CAPES) pelo auxílio financeiro, ao Conselho Nacional de Desenvolvimento Científico e Tecnológico (CNPQ) pela bolsa de produtividade em pesquisa concedida a autora Neiva Leite e a equipe do Laboratório de Investigação em Diabetes e Metabolismo pela colaboração nas análises laboratoriais.

\begin{abstract}
Background: Considered a public health problem, obesity has been cited as a risk factor for the development of asthma. Thus, the overweight has influence on the smooth muscle function and induce systemic inflammation of the airways through the adipocytokines. Although adiponectin is considered an antiinflammatory cytokine, since their concentrations decrease in obesity. The literature has show minimal evidence of this behavior before adipocytokine factors involved in the relationship of obesity and asthma. Thus, the present study aimed to analyze the behavior of adiponectinemia responses and physiological parameters in obese asthmatics and non-asthmatics. Methods: Participated 15 obese adolescents (six asthmatics and nine non-asthmatics), submitted anthropometric measurements, cardiorrespiratory, spirometric and laboratory. The diagnosis of asthma has been through clinical history and ISAAC questionnaire, and obesity as BMI above the $95^{\text {th }}$ percentile. We used the bronchoprovocation test with exercise for assessing exercise-induced bronchospasm (EIB). Results: There was difference in \% Fall FEV $(P=0.034)$ among obese asthmatics and non-asthmatics. For obese asthmatics, the coefficient of determination $\left(R^{2}\right)$ demonstrated effect of adiponectin with SBP $\left(r=-0.512, R^{2}=0.105\right), \mathrm{HDL}\left(r=+0.659, R^{2}=0.292\right)$, glucose $\left(r=-0.599 ; R^{2}=0.199\right)$, glucose120 $\left(r=-0.686, R^{2}=0.338\right)$, insulin120 $\left(r=-0.614, R^{2}=0.221\right)$, Absolute $\mathrm{VO}_{2 \max }\left(\mathrm{r}=+0.518, \mathrm{R}^{2}=0.086\right), \mathrm{VO}_{2 \max }$ Relative FFM $\left(\mathrm{r}=+0.585 ; \mathrm{R}^{2}=0.178\right)$ and \% $\mathrm{FEV} \mathrm{V}_{1}(\mathrm{r}=+0.580$, $\left.R^{2}=0.171\right)$. In obese non-asthmatics were effects of adiponectin with insulin $\left(r=-0.731, R^{2}=0.525\right)$, HOMAIR ( $\left.r=-0.684, R^{2}=0.469\right)$, QUICKI $\left(r=+0.683, R^{2}=0.535\right)$ and $V_{2 \max }$ Relative FFM $\left(r=+0.654, R^{2}=0.346\right)$. Conclusion: There were likely effects of adiponectinemia responses with physiological indicators associated with overweight and low cardiorespiratory fitness in obese asthmatics and non-asthmatics. Furthermore, for the obese asthmatic adiponectinemia demonstrated a possible effect relationship with \% FEV $_{1}$.
\end{abstract}

Key words: Adiponectin. Asthma. Obesity.

\section{Referencias}

1. Janssen I, Katzmarzyk P, Boyce C, Vereecken C, Mulvihill C, Roberts C, Currie C, Pickett W. Comparison of overweight and obesity prevalence in school-aged youth from 34 countries and their relationships with physical activity and dietary patterns. Obes Rev. 2005, 6:123-32.
2. Leite N, Milano GE, Cieslak F, Lopes WA, Rodacki A, Radominski RB. Effects of physical exercise and nutritional guidance on metabolic syndrome in obese adolescents. Rev Bras Fisioter. 2009, 13: 73-81.

3. Oliveira MAB, Leite N. Asma brônquica, doença obstrutiva pulmonar e exercício físico. In: Ghorayeb N, Dioguardi GS. Cardiologia do Esporte e do Exercício. São Paulo: Atheneu, 2007: 443-54. 
4. Shore SA. Obesity and asthma: possible mechanisms. J Allergy Clin Immunol. 2008, 121:1087-93.

5. Poulain M, Doucet M, Major GC, Drapeau V, Series F, Boulet LP, Tremblay A, Maltais F. The effect of obesity on chronic respiratory diseases: pathophysiology and therapeutic strategies. CMAJ. 2006, 174:1293-9.

6. Cieslak F, Lopes WA, Lazarotto L, Timossi LS, Leite N. Parâmetros fisiológicos em adolescents obesos asmáticos e nãoasmáticos submetidos ao broncoespasmo induzido pelo exercício. Motric. 2012; 8(supl.2): 555-66.

7. Kim KW, Shin YH, Lee KE, KIm ES, Sohn MH, Kim KE. Relationship between adipokines and manifestations of childhood asthma. Pediatr Allergy Immunol. 2008; 19: 535-40.

8. Araki S, Dobashi K, Kubo K, Asayama K, Shirahata A. High molecular weight, rather than total, adiponectin levels better reflect metabolic abnormalities associated with childhood obesity. J Clin Endocrinol Metab. 2006; 91: 5113-6.

9. Kraemer RR, Castracane VD. Exercise and humoral mediators of peripheral energy balance: ghrelin and adiponectin. Exp Biol Med.. 2007; 232: 184-94.

10. Kershaw EE, Flier JS. Adipose tissue as an endocrine organ. J Clin Endocrinol Metab. 2004; 89: 2548-56.

11. Balagopal P, George D, Funanage V, Bayne E. Reversal of obesity-related hypoadiponectinemia by lifestyle-intervention: a controlled, randomized study in obese adolescents. J Clin Endocrinol Metab. 2005; 90: 6192-7.

12. Kadowaki T, Yamauchi T. Adiponectin and adiponectina receptors. Endocr Rev. 2005; 26: 439-51.

13. Nemet D, Wang P, Funahashi T, Matsuzawa $Y$, Tanaka $S$, Engelman L, Cooper DM. Adipocytokines, body composition, and fitness in children. Pediatr Res. 2003; 53: 148-52.

14. Bootner A, Kratzsch J, Muller G, Kapellen TM, Bluher S, Keller E, Bluher M, Kiess W. Gender differences of adiponectin levels develop during the progression of puberty and are related to serum androgen levels. J Clin Endocrinol Metab. 2004; 89: 4053-61.

15. Jartti T, Saarikoski L, Jartti L, Lisinen I, Jula A, Huuponen R, Viikari J, Raitakari OT. Obesity, adipokines and asthma. Allergy. 2009; 64: 770-7.

16. Shaibi GQ, Cruz ML, Weigensberg MJ, Toledo-Corral CM, Lane CJ, Kelly LA, Davis JN, Koebnick C, Ventura EE, Roberts CK, Goran MI. Adiponectin indenpendently predicts metabolic syndrome in overweight latino youth. J Clin Endocrinol Metab. 2007; 92: 1809-13.

17. Giouleka P, Papatheodorou G, Lyberopoulos P, Karakatsani A, Alchanatis M, Roussos C, Papiris S, Loukides S. Body mass index is associated with leukotriene inflammation in asthmatics. Eur J Clin Invest. 2007; 92: 2665-71.

18. Field A. Descobrindo a estatística usando o SPSS. Porto Alegre: Artmed, 2009.

19. Kuczmarski RJ, Ogden CL, Guo SS, Grummer-Strawn LM, et al. CDC growth charts: United States. Advance data from vital and health statistics; n. 314. Hyattsville, Maryland: National Center for Health Statistics, 2000.

20. Sociedade Brasileira de Cardiologia. VI Diretrizes Brasileiras de Hipertensão. Arq Bras Cardiol. 2010; 95 (Suppl. 1): 1-51.
21. Tanner JM. Normal growth and techniques of growth assessment. J Clin Endocrinol Metab. 1986; 15: 411-51.

22. Leite N, Milano GE, Lazarotto L, Lopes MFA, Pilotto V, Bento PCB, Cieslak F, Radominski RB. Evaluation and self-assessment of pubertal stage in overweight children and adolescent. Motriz. 2009; 15: 641-7.

23. Houtkooper LB, Going SB, Lohman TG, Roche AF, Van Loan M. Bioelectrical impedance estimation of fat-free body mass in children and youth: a cross-validation study. J Appl Physiol. 1992; 72: 366-73.

24. Rowland TW. Aerobic exercise testing protocols. In: Rowland TW, Varzeus Walsh. Pediatric Laboratory Exercise Testing. Champaign: Human Kinetics Books, 1990.

25. Caiozzo VJ, Davis JA, Ellis JF, Azus JL Vandagriff R, Prietto CA, Mcmaster WC. A comparison of gas exchange indices used to detect the anaerobic threshold. J Appl Physiol. 1982; 53: 1184-9.

26. Sociedade Brasileira de Pneumologia e Tisiologia. IV Diretrizes da Sociedade Brasileira de Pneumologia e Tisiologia para o Manejo da Asma. J Bras. Pneumol. 2012; 38 (supl.1): 1-46.

27. Polgar $\mathrm{C}$, Promodhat V. Pulmonary function testing in children: techniques and standards. Philadelphia: WB Saunders Co., 1971.

28. Sociedade Brasileira De Pneumologia E Tisiologia. Diretrizes para Teste de Função Pulmonar. J Pneumol. 2002; 28: 1-238.

29. Friedwald WT, Levy RI, Fredrickson D. Estimation of the concentration of low-density lipoprotein cholesterol in plasma without use of the preparative ultracentrifugue. Clin Chem. 1972; 18: 499-502.

30. Sociedade Brasileira De Cardiologia. I Diretriz de prevenção de aterosclerose na infância e adolescência. Arq Bras Cardiol. 2005; 85: 1-36.

31. American Diabetes Association. Diagnosis and classification of Diabetes Mellitus. Diabetes Care. 2006; 29: 43-8.

32. Matthews DR, Hosker JP, Rudenski AS, Naylor BA, Treacher DF, Turner RC. Homeostasis model assessment: insulin resistance and beta cell-function from fasting plasma glucose and insulin concentrations in man. Diabetologia. 1985; 28 : 412-9.

33. Katz A, Nambi SS, Mather K, Baron AD, Follmann DA, Sullivan G, Quon MJ. Quantitative insulin sensitivity check index: a simple, accurate method for assessing insulin sensitivity in humans. J Clinical Endocrinol Metab. 2000, 85: 2402-10.

34. Martinez EC, Fortes MSA, Anjos LA. Influência do estado nutricional e do $\mathrm{VO}_{2 \max }$ nos níveis de adiponectina em homens acima de 35 anos. Arq Bras Cardiol. 2011; 96: 471-6.

35. Rodrigues JC, Takahashi A, Olmos FMA, Souza JB, Bussamra MHF, Cardieri JMA. Efeito do índice de massa corpórea na gravidade da asma e na reatividade brônquica induzida pelo exercício em crianças asmáticas com sobrepeso e obesas. Rev Paul Pediatr. 2007; 25: 207-13.

36. Ulger Z, Demir E, Tanac R, Goksen D, Gulen F, Darcan S, Can $D$, Coker M. The effect of childhood obesity on respiratory function tests and airway hyperresponsiveness. Turk $\mathrm{J}$ Pediatr. 2006; 48: 43-50. 